\title{
759Temporal tuning in the acquisition of cognitive skill
}

\author{
RICHARD A. CARLSON and LISA M. STEVENSON \\ Pennsylvania State University, University Park, Pennsylvania
}

\begin{abstract}
The temporal tuning hypothesis suggests that individuals adjust the timing of cognitive performances to achieve temporal coordination of mental processes and the data on which they operate, and that this adjustment becomes more precise with practice. Participants in two experiments performed self-paced multiple-step arithmetic tasks in which the information needed for each step was briefly displayed at the participants' request. Timing constraints were manipulated by varying between subjects the delay between requests and displays of information. In Experiment 1, both operators and operands appeared step by step, and participants achieved a modest degree of temporal adjustment that did not change with practice. In Experiment 2, participants could preview operators while operands appeared step by step. In that experiment, participants achieved more precise temporal adjustment, and the amount of adjustment increased with practice. These results demonstrate the phenomenon of temporal tuning in symbolic cognitive skills and suggest some constraints on the ability to anticipate the time course of one's mental processes.
\end{abstract}

With practice, cognitive skills become faster and more fluent. Theorists have proposed two mechanisms to account for increasing fluency: speedup of component processes, and restructuring that produces more efficient procedures. Another possible mechanism for increased fluency is what we call temporal tuning, the adjustment of cognitive processes to improve the coordination of mental operations with the availability of the information on which they operate.

The need for temporal coordination is obvious, and the phenomenon has been extensively studied (e.g., Rosenbaum \& Collyer, 1998) in the case of perceptual-motor skills. For example, consider catching a thrown ball. Successful performance of this task requires that movements of the arm and hand be coordinated both spatially and temporally with the flight of the ball, and the success of this coordination depends on practice. Given the view that cognitive and perceptual-motor skills are essentially similar (Carlson, 1997; Rosenbaum, Carlson, \& Gilmore, 2001), it is reasonable to assume that similar temporal coordination may be necessary in cognitive tasks. We consider an analogue of the temporal coordination aspect in a symbolic cognitive task, mental arithmetic. In mental arithmetic, coordination involves selecting operands just as a mental procedure for calculation is ready to process them.

We thank Lori Forlizzi, Marc Grosjean, Tim Rickard, Mike Stadler, and an anonymous reviewer for their constructive comments on earlier drafts. We are grateful to Rickard and Stadler for suggesting the analysis including experiment as a factor. Portions of these data were presented in a poster at the 22nd Annual Meeting of the Cognitive Science Society, Philadelphia, August 13, 2000. Correspondence concerning this article should be addressed to R. A. Carlson, Department of Psychology, Pennsylvania State University, 613 Moore Building, University Park, PA 16802-3106(e-mail: racarlson@psu.edu).
Fluent cognitive performance depends on concurrent activation of appropriate representations of goals, procedures, and data. This observation is reflected in a number of current theories, such as ACT-R (Anderson \& Lebiere, 1998). Together with the observation that improvement of many skills with practice can be attributed to better coordination of actions with the environment (e.g., Neisser, 1983), this suggests the temporal tuning hypothesis: As a consequence of practice, individuals will learn to adjust the timing of perceptual and memory retrieval processes to make information available at times that allow optimal integration with ongoing mental activity. This hypothesis makes the two specific predictions examined here: Individuals will pace their performance to compensate for externally imposed temporal constraints on the availability of information, and this compensation will become more precise with practice. These predictions assume that individuals are able, or can learn, to anticipate the time course of their own mental processes as they perform cognitive tasks. For example, learners may identify internal cues that serve as markers for the progress of mental processes and can thus serve as timing cues for subsequent processes. Thus learners in a serial reaction time (SRT) task might use internal shifts of attention as cues for timing responses and subsequent shifts of attention, as suggested by research indicating that secondary tasks interfere with SRT performance by disrupting the timing of the primary task (Dominey, 1998; Stadler, 1995).

The present study examines temporal tuning in the context of running arithmetic. Previous research suggests that the operator-operand structure of conventional arithmetic notation also characterizes the mental processes involved. For example, individuals perform mental arithmetic more fluently when operators are availablein advance of operands, 
in both single-step and sequential multiple-step tasks (Carlson \& Sohn, 2000; Sohn \& Carlson, 1998). Previous research has demonstrated the learning of timing constraints in perceptual-motor (Dominey, 1998) and cognitive tasks (Carlson, Shin, \& Wenger, 1994). Grosjean, Rosenbaum, and Elsinger (2001) have recently demonstrated that individuals learn timing in repetitive choice reaction time tasks, producing increased error rates or slowed responding when displays appear earlier or later than expected. Research on skilled anticipation (e.g., Rowe \& McKenna, 2001) suggests that expertise in a variety of real-world tasks is characterized by the acquired ability to anticipate the time course of events. Gopher, Armony, and Greenshpan (2000) have demonstrated that preparation time at the beginning of blocks in a taskswitching paradigm depended on control requirements, and Hübner, Futterer, and Steinhauser (2001) have demonstrated a similar effect on the basis of trial-by-trial cues specifying the upcoming task, suggesting that individuals are sensitive to the temporal requirements of mental operations. The contribution of the present study is to examine the learning of temporal constraints when compensating for those constraints depends on anticipating when one will be ready to use a piece of information to complete a cognitive task.

\section{Experimental Task}

Participants performed a multiple-step running arithmetic task used in previous research (e.g., Carlson \& Sohn, 2000) and presented on a desktop computer. At each of eight steps, the participant updated the running total on the basis of an arithmetic operation and a new operand. The information needed to perform each step appeared briefly, after the participant requested it by pressing the space bar on the computer keyboard. In Experiment 1, both the operator and the operand appeared step by step; in Experiment 2 , the series of operators was visible throughout each problem, and operands appeared step by step. Figure 1 illustrates the displays.

To test the temporal tuning hypothesis, we introduced temporal constraints by inserting delays between participants' requests for information and the display of that information. For some participants, the delay was brief $(200 \mathrm{msec})$; for others, the delay represented a substantial proportion of the time that they needed to perform each step $(1,100 \mathrm{msec})$. In Experiment 1, a third group of participants had an intermediate delay of $650 \mathrm{msec}$. At each step information appeared only briefly $(200 \mathrm{msec})$, followed by a mask, so participants had to pick up the information at a fairly precise point in time.

Three possible outcomes have different implications for the temporal tuning hypothesis. One possibility is that participants will not be able to compensate for longer delays, so that there will be no difference in request times as a function of delay. For example, problem-solving steps might be realized by procedures with inaccessible internal structures that do not provide a basis for anticipation or for control of timing. If so, requesting information for an upcoming step might have to wait for completion of the current step. This possibility would be reflected in a null effect of delay length. We expected, however, that participants in all delay conditions would perform faster with increasing practice, as demonstrated in previous studies of running arithmetic (e.g., Carlson \& Lundy, 1992).

A second possibility is that individuals will strategically adjust the timing of their requests for information on the basis of perception of the delay, but not tune this adjustment as a function of practice. If this is the case, adjustments to delay length should occur very quickly (because the long delays used here are very salient) and change little as a function of practice. This possibility would be reflected in a main effect of delay length, with little or no interaction of delay and practice. Optimal adjustment would result in latencies for longer delay groups that compensate completely for the additional delay relative to the shortdelay baseline. However, strategic adjustment might not be optimal, because it might not take into account the time course of mental processes that themselves speed up with practice.

A third possibility, consistent with the temporal tuning hypothesis, is that with practice participants will learn to anticipate when they will be ready for the new information and time their requests for information while taking into account the delay. This possibility would be reflected in latencies for longer delay groups that increasingly approach optimal compensation with greater practice. Delay length and practice should thus have interacting effects on request time, with request times decreasing more with practice for longer delays. This possibility is not incompatible with strategic adjustment, in the sense that learners might make strategic adjustments quickly, then tune those adjustments with practice. It is the predicted interaction of delay with practice, however, that distinguishes the temporal tuning hypothesis from the hypothesis that learners make only strategic adjustments on the basis of perception of the delay.

\section{EXPERIMENT 1}

The participants in Experiment 1 performed the selfpaced sequential arithmetic task described above, with one of three delays imposed between each request for information and the display of that information.

\section{Method}

Task and equipment. Participants solved eight-step running arithmetic problems as described above. Each problem included two steps with each of four operations: ADD, DIF, MIN, and MAX. ADD required participants to replace their current total with the sum of the previous total and the new operand. DIF represented unsigned subtraction; participants replaced their current total with the absolute value of the difference between the current total and the new operand. MIN and MAX required participants to compare their current total with a new operand, retaining as the new total either the smaller (MIN) or the larger (MAX) of the current total and the new operand. This set of operations has been used successfully in several previous studies (e.g., Carlson \& Lundy, 1992; Wenger \& Carlson, 1996), and participants had no difficulty in understanding and applying them.

At each step, the participant's keypress request for information was followed by the appropriate delay; then the operator was dis- 


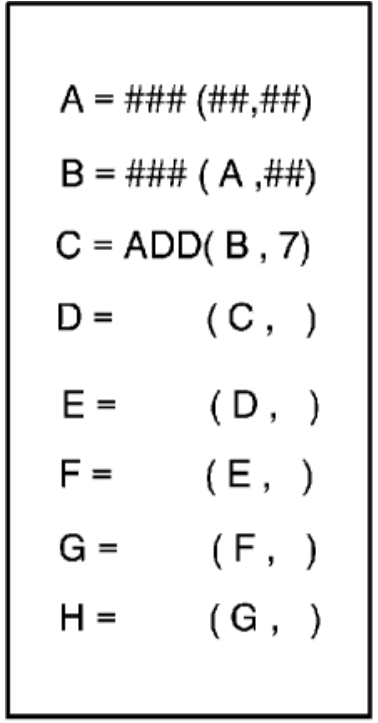

Experiment 1

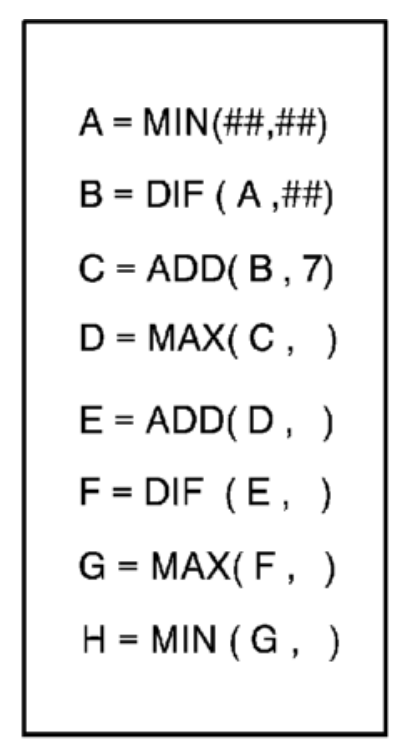

Experiment 2
Figure 1. Sample task displays following a participant's request for information on Step 3. The left panel exemplifies the displays used in Experiment 1, and the right panel the displays used in Experiment 2.

played for $200 \mathrm{msec}$. The operator was then masked, and $200 \mathrm{msec}$ later the operand was displayed. After an additional $200 \mathrm{msec}$, the operand display was masked. Masks remained on the display for the remainder of the problem (see Figure 1).

Displays and response collection were controlled by IBM-compatible microcomputers, programmed using the MEL software from Psychology Software Tools.

Design and Procedure. Participants practiced solving eight-step problems for eight blocks of 10 trials. In the first block, the delay between request and display was the same $(650 \mathrm{msec})$ for all participants. On Blocks 2-8, the between-subjects manipulation of delay was introduced. Three groups of participants experienced delays of 200,650 , or $1,100 \mathrm{msec}$, respectively between their space-bar responses and the onset of the operator/operand display.

Participants. Thirty-six undergraduate students participated in return for a small amount of course credit. Twelve participants were assigned to each of the 3 groups.

\section{Results and Discussion}

The data include accuracy of the final responses for each problem and step-by-step request latencies measured from the onset of the operand for each step. We considered separately the latency for the first step, because two new operands appeared at that step, and for the last (eighth) step, because no new operand appeared in response to that keypress. Median latency for Steps 2-7 was calculated for each problem that a participant answered correctly, and served as a dependent variable for the most central analyses. Figure 2 summarizes these median latency data for correct trials.

In Block 1, before the experimental manipulation was introduced, the overall mean proportion correct was .77, and accuracy did not differ among conditions $[F(2,33)=$ $\left.1.21, M S_{\mathrm{e}}=0.779, p>.3\right]$. Latencies for the first step $(M=1,582 \mathrm{msec})$ and last step $(M=1,453 \mathrm{msec})$ and median latency for Steps $2-7(M=1,125 \mathrm{msec})$ also did not differ among conditions (all $F \mathrm{~s}>1$ ).

In Blocks 2-8, the experimental manipulation of delay had an effect on accuracy $\left[F(2,33)=3.78, M S_{\mathrm{e}}=2.291\right.$, $p=.03$ ]. Mean proportion correct was $.76, .85$, and .86 for the 200-, 650-, and 1,100-msec delay groups, respectively. Practice block had no significant effect on accuracy and did not interact with delay condition (both $F_{\mathrm{S}}>1$ ).

Delay condition and practice block both had effects on first-step latency, last-step latency, and median latency for Steps 2-7. Figure 2 shows these effects for median latency, and similar patterns were observed for first-step and last-step latencies. Inferential statistics are summa-

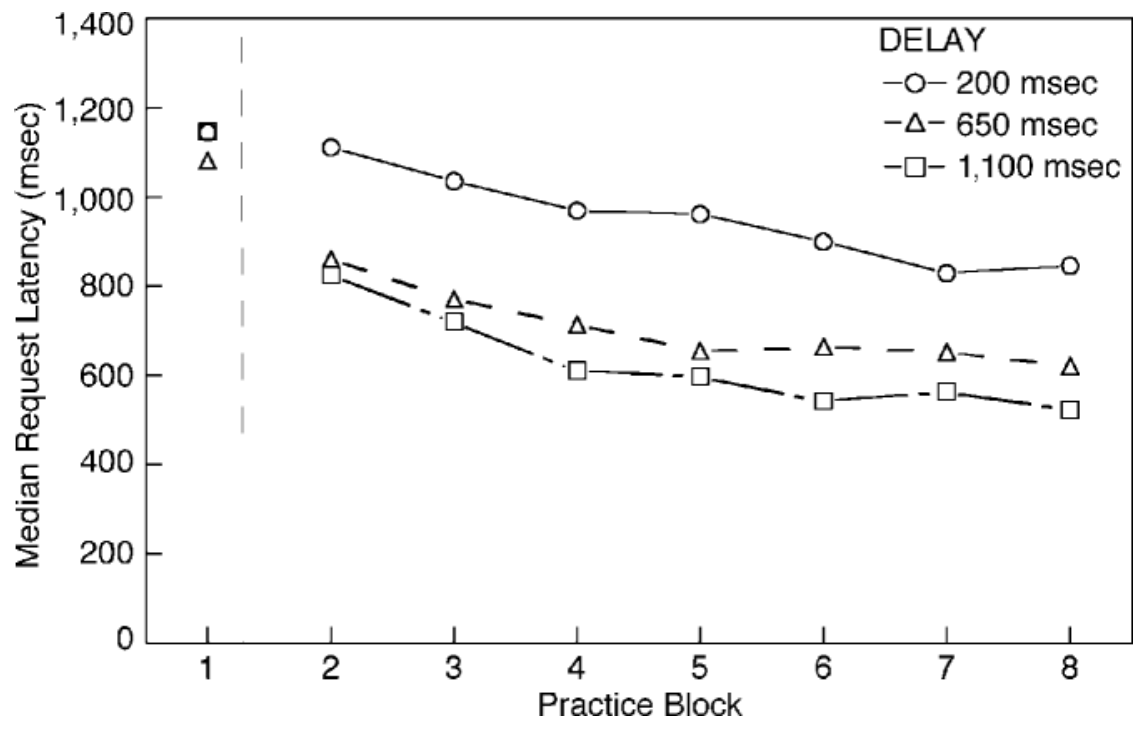

Figure 2. Median request times on problem Steps 2-7, Experiment 1. 
rized in Table 1. As expected, participants with longer delays between request and display responded more quickly, partially compensating for the difference in delays. On Steps 2-7 in the final practice block, participants in the 650 -msec delay condition requested information an average of $226 \mathrm{msec}$ more quickly than those in the 200-msec delay condition, and those in the 1,100-msec delay condition made their requests an average of $322 \mathrm{msec}$ earlier. The observed adjustment of timing is clearly suboptimal; for example, optimal adjustment to the 1,100-msec delay would result in request times $900 \mathrm{msec}$ shorter than those observed in the 200-msec delay condition. Furthermore, for none of these measures was there a significant interaction between delay and practice block, indicating no substantial improvement in temporal adjustment with practice. The modest amount of temporal adjustment and lack of improvement with practice suggest that participants adopted deliberate strategies in response to the delays early in practice, and did not change those strategies as predicted by the temporal tuning hypothesis.

\section{EXPERIMENT 2}

The participants in Experiment 1 demonstrated some sensitivity to temporal constraints, but the effect of delay was relatively small and did not change with practice, suggesting a deliberate strategic adjustment rather than temporal tuning. In Experiment 2, we examined the hypothesis that greater precision of temporal adjustment with practice would be possible when learners could preview upcoming operators. Previous research has demonstrated greater fluency in performing similar multiple-step tasks when operator sequences are consistent from problem to problem (Carlson \& Lundy, 1992), or when the operator sequence is displayed throughout the problem to allow preview (Carlson \& Shin, 1996). Similar benefits of preview have been demonstrated in sequential perceptual-motor tasks (e.g., Pashler, 1994; Salthouse, 1986; Wright, 1990).

A theoretical justification for this hypothesis can be developed in terms of Anderson and Lebiere's (1998) ACT-R production-system model. Although the instantiation of a goal as an active intention corresponds to the selection of a particular production for execution, advance activation of declarative representations of upcoming operations should make this process more fluent. ACT-R does not include obvious mechanisms for temporal tuning, but the relation between declarative and procedural knowledge embodied in that theory allows for benefits of operator preview.
Participants in Experiment 2 solved problems identical to those in Experiment 1, with the exception that the operators for all steps were continuously visible throughout each problem. Space-bar requests for information thus controlled the display of new operands for each step. In Experiment 2 , the delay between request and display was either 200 or $1,100 \mathrm{msec}$, and each new operand was displayed for $200 \mathrm{msec}$, followed by a mask.

\section{Method}

Except as noted above, the task, equipment, design, and procedure were identical to those used in Experiment 1.

Participants. Twenty-five undergraduate students participated in exchange for a small amount of extra course credit. Random assignment resulted in 13 participants in the 200-msec delay condition and 12 participants in the 1,100 -msec delay condition.

\section{Results and Discussion}

We analyzed the same dependent variables examined in Experiment 1. Figure 3 summarizes the median latency data.

In Block 1, before the experimental manipulation was introduced, mean proportion correct was .89 and did not differ between groups $[t(23)=1.61, p>.1]$. Participants in the 1,100-msec delay condition had somewhat shorter latencies on the first $(M=1,593 \mathrm{msec})$ and last $(M=$ $1,458 \mathrm{msec}$ ) problem steps than did those in the 200 -msec delay condition $(M=1,812$ and $1,630 \mathrm{msec}$, respectively), but neither difference approached significance $[t(23)=1.04$ and 0.91]. A similar difference was observed for median request time on problem Steps 2-7, but this effect also did not approach significance $[t(23)=$ 1.16]. The mean of median request times was $1,168 \mathrm{msec}$ for the 1,100-msec delay group and $1,319 \mathrm{msec}$ for the 200 -msec delay group. Note that these times represent faster performance than in Experiment 1. The total median time per step including delay and display times for operator and mask in Block 1 of Experiment 1 was $2,175 \mathrm{msec}$, whereas the delay and request times here encompass the entire step time $(M=1,897 \mathrm{msec})$.

Mean proportion correct was .89 for Blocks $2-8$. There was a marginally significant effect of block $[F(6,138)=$ 2.03, $M S_{\mathrm{e}}=0.0090, p=.065$ ], reflecting primarily a small increase in accuracy over the first few blocks. Neither the main effect of delay condition nor its interaction with block approached significance $\left(F_{\mathrm{S}}>1\right)$.

Delay condition and practice block again had significant effects (Table 2) on each of the latency measures, as shown in Figure 3. Two features of these data are especially noteworthy. First, in contrast the results of Experi-

Table 1

Inferential Statistics for Latency Analyses, Experiment 1 Practice Blocks

\begin{tabular}{llll}
\hline & \multicolumn{3}{c}{ Dependent Variable } \\
\cline { 2 - 4 } Effect & \multicolumn{1}{c}{ First-Step Latency } & \multicolumn{1}{c}{ Last-Step Latency } & Median Latency Steps 2-7 \\
\hline Block & $F(6,198)=8.8, M S_{\mathrm{e}}=5,495,823, p>.001$ & $F(6,198)=22.4, M S_{\mathrm{e}}=10,326,791, p>.001$ & $F(6,198)=33.8, M S_{\mathrm{e}}=1,969,574, p>.001$ \\
Delay & $F(2,33)=4.7, M S_{\mathrm{e}}=391,720, p=.016$ & $F(2,33)=4.7, M S_{\mathrm{e}}=391,720, p=.016$ & $F(2,33)=5.8, M S_{\mathrm{e}}=414,368, p>.01$ \\
$\begin{array}{l}\text { Block } \times \\
\text { delay }\end{array}$ & $F(12,198)=0.7, M S_{\mathrm{e}}=5,495,823, p>.5$ & $F(12,198)=1.4, M S_{\mathrm{e}}=10,326,791, p=.18$ & $F(12,198)=0.8, M S_{\mathrm{e}}=1,969,574, p>.5$ \\
\hline
\end{tabular}




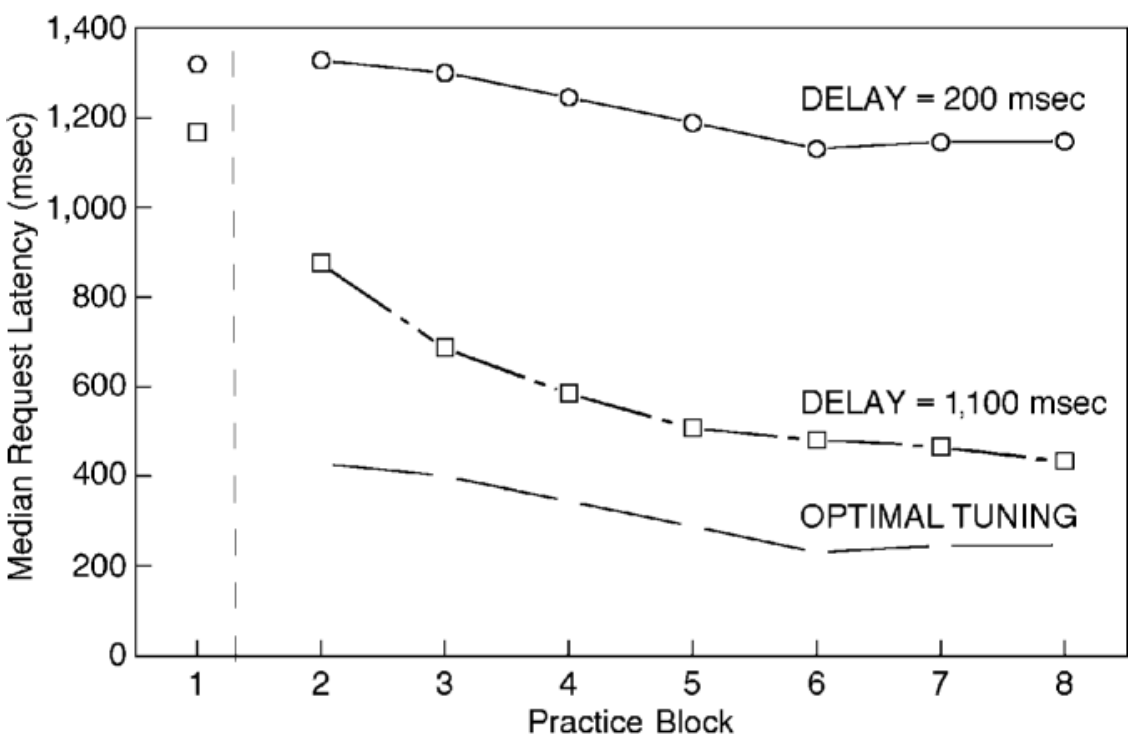

Figure 3. Median request times on problem Steps 2-7, Experiment 2.

ment 1 , the difference between delay conditions in median latency for problem Steps 2-7 increased significantly with practice, from $452 \mathrm{msec}$ in Block 2 to $713 \mathrm{msec}$ in Block 8 . Second, the magnitude of the delay effect was much greater than that observed in Experiment 1. Although participants in the long-delay condition did not achieve optimal adjustment, their request times clearly approach optimal compensation with practice. This is illustrated by the convergence of these participants' data to the curve at the bottom of Figure 3, which reflects optimal compensation as determined by subtracting $900 \mathrm{msec}$ from the baseline request times observed in the 200-msec delay condition, reflecting the timing difference in the two delay conditions. Optimal compensation would suggest that participants in the long delay condition were completely compensating for the 900 -msec delay difference $(1,100 \mathrm{msec}$ for the longdelay condition minus $200 \mathrm{msec}$ for the short-delay condition). The particular optimal time to make a response changed over time, reflecting the finding that participants in both delay conditions performed faster with increasing practice, replicating previous studies of running arithmetic (e.g., Carlson \& Lundy, 1992).

One possibility is that the averaging over participants obscured individual differences in adjustment times. It could be, for example, that each participant in the longdelay condition made a strategic adjustment, but the dif- ferential timing of these adjustments makes it appear as though the learning was gradual. A visual inspection of learning curves for individual participants suggests that this is not the case. All but 2 participants began to show some improvement between the second and third blocks (the first two blocks of practice with the long delay) and continued to improve with practice. The 2 participants who failed to show significant improvement averaged an improvement of $4.6 \mathrm{msec}$ between these blocks, as opposed to an average of $224 \mathrm{msec}$ for all other participants (ranging from $66 \mathrm{msec}$ to $453 \mathrm{msec}$ ). These 2 participants were removed from further data analysis to examine the possibility that their later adjustment might combine with other results in a way that obscured strategic adjustment. This did not change the overall pattern of results. In fact, the evidence for the practice $\times$ condition interaction was even stronger $\left[F(1,21)=7.77, M S_{\mathrm{e}}=223,826, p=.01\right]$. This suggests that temporal tuning was occurring in Experiment 2 , rather than simply strategic adjustment occurring at different times for different participants.

To further examine the idea that temporal tuning was taking place in Experiment 2 and not in Experiment 1, we conducted an analysis of variance including experiment as a factor, again excluding the 2 participants in Experiment 2 who did not show improvement between the second and third training blocks. The three-way interaction

Table 2

Inferential Statistics for Latency Analyses, Experiment 2 Practice Blocks

\begin{tabular}{llll}
\hline & \multicolumn{3}{c}{ Dependent Variable } \\
\cline { 2 - 4 } Effect & \multicolumn{1}{c}{ First-Step Latency } & \multicolumn{1}{c}{ Last-Step Latency } & Median Latency Steps 2-7 \\
\hline Block & $F(6,138)=6.2, M S_{\mathrm{e}}=11,642,731, p>.001$ & $F(6,138)=17.8, M S_{\mathrm{e}}=6,987,440, p>.001$ & $F(6,138)=26.5, M S_{\mathrm{e}}=1,773,860, p>.001$ \\
Delay & $F(1,23)=16.5, M S_{\mathrm{e}}=977,537, p=.016$ & $F(1,23)=20.6, M S_{\mathrm{e}}=484,164, p>.001$ & $F(1,23)=60.3, M S_{\mathrm{e}}=292,829, p>.001$ \\
Block $\times$ & & & \\
delay & $F(6,138)=1.2, M S_{\mathrm{e}}=11,642,731, p>.3$ & $F(12,138)=1.0, M S_{\mathrm{e}}=6,987,440, p>.4$ & $F(12,138)=3.6, M S_{\mathrm{e}}=1,773,860, p=.002$ \\
\hline
\end{tabular}


was significant $\left[F(6,258)=2.24, M S_{\mathrm{e}}=26,524, p=\right.$ .04]. As described previously, an interaction between block and delay was seen in Experiment 2 but not in Experiment 1 , consistent with the conclusion that temporal tuning depends on the opportunity for operator preview provided in Experiment 2.

\section{GENERAL DISCUSSION}

These results demonstrate temporal tuning in the performance of a symbolic cognitive task. Participants adjusted the timing of their requests for information to compensate for externally imposed delays. Given operator preview, individuals are very good at anticipating when they will be ready to process operands. However, when they attempt to anticipate when they will be ready for information specifying the next goal to be instantiated, they are much less successful, as is shown by the smaller temporal adjustment observed in Experiment 1 when operators appeared step by step.

Both experiments provide evidence of adjustment to temporal constraints: Participants in conditions with longer delays requested information earlier than did those in conditions with shorter delays. Speeded anticipation seems to characterize expert performance in sports and other dynamic perceptual-motor activities (e.g., Rowe \& McKenna, 2001). Our data suggest that people can similarly learn to anticipate the timing of their own mental activity, at least under some circumstances. When operators for all steps were continuously visible (Experiment 2), participants were able to anticipate the timing more precisely and improve that anticipation with practice, consistent with the temporal tuning hypothesis. This difference between experiments suggests constraints on the ability to anticipate and control the timing of mental activity. For example, it may be that to apply a procedure, a goal must be instantiated to provide a basis for precise anticipatory timing, perhaps allowing temporal tuning by providing a reference point for the timing of mental processes that implement that goal. In Experiment 2, information specifying the goal of a step could be picked up, perhaps allowing the goal to be instantiated, at any point during the execution of the previous step. For example, it might be that individuals could consider the goal of the next step while awaiting the result of the current step (Carlson, 1997). One theoretical account of this possibility is that instantiating a goal evokes a procedural frame or schema that might include timing information (Carlson \& Sohn, 2000). Such overlapping of steps may not have been possible in Experiment 1 because participants did not have control of the relative timing of the operator and the new operand, therefore precluding more precise tuning. It is also possible that the greater difficulty of the no-preview version of the task (as shown by somewhat lower accuracy in Experiment 1) made it harder for participants to adjust the timing of their performance, but of course the greater difficulty of the nopreview case may result in part from the inability of participants to optimally sequence and time the components of their performance under those circumstances.
Participants in all conditions of both experiments performed faster as a result of practice. Although simple arithmetic is a well-learned skill for college students, practice likely results in both speed-up of individual operations (e.g., Rickard, Healy, \& Bourne, 1994) and learning of other aspects of the running arithmetic task (e.g., Wenger \& Carlson, 1996). It is possible that some of this general speedup is due to temporal fine-tuning at a fine grain of cognitive procedures not revealed by the present methodology, as suggested by Neisser (1983). For example, practice might result in the tuning of procedures to the temporal characteristics (rise and decay times) of activation of intermediate results in memory.

Are our findings unique to mental arithmetic, which is characterized by an operator-operand structure codified in conventional notation? Carlson and Sohn (2000) considered this issue for the operator-first benefit, demonstrating parallel results with arithmetic and spatial tasks. In pilot research, we have found results parallel to those of Experiment 1 with a spatial task; however, we have not yet attempted to replicate the more important results of Experiment 2 in a nonarithmetic task. However, we expect that temporal tuning as a consequence of practice is a widespread phenomenon because we believe that the need for temporal coordination of procedures and data is a general characteristic of cognitive skill.

Some recent related research has also examined the time course of intentions, particularly with respect to task switching. At least under some circumstances, it appears that participants can effectively prepare for a task switch, as indicated by reduced switch costs, when given an advanced cue about the task (Gopher et al., 2000; Hübner et al., 2001). Furthermore, external rather than internal cues appear to be more effective in some cases (e.g., Rogers \& Monsell, 1995). The contrast between Experiments 1 and 2 is consistent with these general ideas, although the specific issues are somewhat different.

Given the view that cognitive and perceptual-motor skills are essentially similar (Carlson, 1997; Rosenbaum et al., 2001), it seems plausible that the use of "lookahead" strategies to control timing is a general feature of skilled performance. In the perceptual-motor domain, vision provides information specifying affordances available in the near future (e.g., looking ahead while walking). An interesting possible implication of these findings is that the benefits of consistent sequences of operators or subgoals in mental tasks (Carlson \& Lundy, 1992; Wenger $\&$ Carlson, 1996) are mediated by temporal tuning. The ability to anticipate upcoming processes may allow for more precise juxtaposition of procedures and operands, resulting in the increased fluency observed in consistent sequence relative to varied sequence conditions. Precise juxtaposition minimizes the need for working memory management and the coordination of information from working memory and perception, which are demanding and time consuming (e.g., Carlson, 1997; Carlson, Wenger, $\&$ Sullivan, 1993).

We have not considered detailed models for temporal tuning. A model that integrated the goal-driven processing 
of production-system models (e.g., Anderson \& Lebiere, 1998) with information-processing mechanisms for the control of timing (e.g., Grosjean et al., 2001; Rosenbaum, 1998) might account for this phenomenon. Such a model might integrate work on the timing of perceptual-motor skills with mechanisms that capture the operator-operand structure of symbolic cognitive skills. The present results suggest that Neisser's (1983) proposal is correct, and that temporal tuning may be an important aspect of cognitive skill.

\section{REFERENCES}

Anderson, J. R., \& Lebiere, C. (1998). The atomic components of thought. Mahwah, NJ: Erlbaum.

CARlson, R. A. (1997). Experienced cognition. Mahwah, NJ: Erlbaum.

Carlson, R. A., \& Lundy, D. H. (1992). Consistency and restructuring in learning cognitive procedural sequences. Journal of Experimental Psychology: Learning, Memory, \& Cognition, 18, 127-141.

Carlson, R. A., \& ShIn, J. C. (1996). Practice schedules and subgoal instantiation in cascaded problem solving. Journal of Experimental Psychology: Learning, Memory, \& Cognition, 22, 157-168.

Carlson, R. A., Shin, J. C., \& Wenger, J. L. (1994, November 12). Timing and the control of fluent cognitive sequences. Paper presented at the 35th annual meeting of the Psychonomic Society, St. Louis.

CArlson, R. A., \& Sohn, M.-H. (2000). Cognitive control of multistep routines: Information processing and conscious intentions. In S. Monsell \& J. Driver (Eds.), Control of cognitive processes: Attention and performance XVIII (pp. 443-464). Cambridge, MA: MIT Press.

Carlson, R. A., Wenger, J. L., \& Sullivan, M. A. (1993). Coordinating information from perception and working memory. Journal of Experimental Psychology: Human Perception \& Performance, 19, 531548.

Dominey, P. F. (1998). Influences of temporal organization on sequence learning and transfer: Comments on Stadler (1995) and Curran and Keele (1995). Journal of Experimental Psychology: Learning, Memory, \& Cognition, 24, 234-248.

Gopher, D., Armony, L., \& Greenshran, Y. (2000). Switching tasks and attention policies. Journal of Experimental Psychology: General, 129, 308-339.

Grosjean, M., Rosenbaum, D. A., \& Elsinger, C. (2001). Timing and reaction time. Journal of Experimental Psychology: General, 130, 256-272.

Hübner, R., Futterer, T., \& Steinhauser, M. (2001). On attentional control as a source of residual shift costs: Evidence from two-component task shifts. Journal of Experimental Psychology: Learning, Memory, \& Cognition, 27, 640-653.

NeIsser, U. (1983). Toward a skillful psychology. In D. R. Rogers \& J. A. Sloboda(Eds.), Acquisition of symbolic skill (pp. 1-17). New York: Plenum.

PASHLER, H. (1994). Overlapping mental operations in serial performance with preview. Quarterly Journal of Experimental Psychology, 47A, 161-191.

Rickard, T. C., Healy, A. F., \& Bourne, L. E., JR. (1994). On the cognitive structure of basic arithmetic skills: Operation, order, and symbol transfer effects. Journal of Experimental Psychology: Learning, Memory, \& Cognition, 20, 1139-1153.

Rogers, R. D., \& Monsell, S. (1995). Costs of a predictable switch between simple cognitive tasks. Journal of Experimental Psychology: General, 124, 207-231.

Rosenbaum, D. A. (1998). Broadcast theory of timing. In D. A. Rosenbaum \& C. E. Collyer (Eds.), Timing of behavior: Neural, psychological, and computationalperspectives (pp. 215-235). Cambridge, MA: MIT Press.

Rosenbaum, D. A., Carlson, R. A., \& Gilmore, R. O. (2001). Acquisition of intellectual and perceptual-motor skills. Annual Review of Psychology, 52, 453-470.

Rosenbaum, D. A., \& Collyer, C. E. (Eds.) (1998). Timing ofbehavior: Neural, psychological, and computational perspectives. Cambridge, MA: MIT Press.

Rowe, R. M., \& McKenna, F. P. (2001). Skilled anticipation in realworld tasks: Measurement of attentional demands in the domain of tennis. Journal of Experimental Psychology: Applied, 7, 60-67.

Salthouse, T. A. (1986). Perceptual, cognitive, and motoric aspects of transcription typing. Psychological Bulletin, 99, 303-319.

SoHn, M.-H., \& CARLSON, R. A. (1998). Procedural frameworks for simple arithmetic skills. Journal of Experimental Psychology: Learning, Memory, \& Cognition, 24, 1052-1067.

STADLER, M. A. (1995). Role of attention in implicit learning. Journal of Experimental Psychology: Learning, Memory, \& Cognition, 21, 674-685.

Wenger, J. L., \& CARlson, R. A. (1996). Cognitive sequence knowledge: What is learned? Journal of Experimental Psychology: Learning, Memory, \& Cognition, 22, 599-619.

Wright, C. E. (1990). Controlling sequential motor activity. In D. N. Osherson, S. M. Kosslyn, \& J. M. Hollerbach (Eds.), Visual cognition and action: An invitation to cognitive science (pp. 285-316). Cambridge, MA: MIT Press.

(Manuscript received January 23, 2001; revision accepted for publication November 29, 2001.) 